\title{
CONTINUOUS PROGRAMMING CONTAINING ARBITRARY NORMS
}

\author{
S. CHANDRA, B. D. CRAVEN and I. HUSAIN
}

(Received 13 December 1983)

Communicated by B. Mond

\begin{abstract}
Optimality conditions and duality results are obtained for a class of cone constrained continuous programming problems having terms with arbitrary norms in the objective and constraint functions. The proofs are based on a Fritz John theorem for constrained optimization in abstract spaces. Duality results for a fractional analogue of such continuous programming problems are indicated and a nondifferentiable mathematical programming duality result, not explicitly reported in the literature, is deduced as a special case.
\end{abstract}

1980 Mathematics subject classification (Amer. Math. Soc.): 90 C 48, 90 C 30.

\section{Introduction}

A detailed study of the duality aspects of a class of constrained variational problems has been presented by Mond and Hanson [22]. Recently a number of duality theorems for different forms of continuous programming or control problems have appeared in the literature, notably by Rockafellar [28, 29, 30], Abrham and Buie [1, 2, 3], Reiland [25], Reiland and Hanson [26] and other references cited in these papers.

The present authors in [6] studied duality aspects of a nondifferentiable analogue of the problem treated in [22], the nondifferentiability entering due to the square root of a quadratic form appearing in the integrand of the objective functional. In this paper, optimality conditions and duality results for a more general class of continuous programming problems are given. These continuous

(C) 1985 Australian Mathematical Society $0263-6115 / 85 \$ A 2.00+0.00$ 
programming problems are cone constrained and contain terms with arbitrary norms of linear functions in the objective and constraint functions. The dual problem considered is a modified Wolfe dual rather than a Lagrangian or conjugate convex dual considered by some other authors.

Here the problem is expressed very directly as a mathematical programming problem in function spaces and then a Fritz John theorem [15] for constrained optimization is applied. This approach readily leads to duality and converse duality. In this context it is remarked that optimal control results of Clarke $[8,9]$ could be used, instead of the Fritz John theorem of [15], leading to similar formulas under somewhat different hypothesis. However, nontrivial questions of representing subdifferentials arise with either approach. Also the present approach could allow some weakening of the convexing hypothesis along the lines of Weir, Hanson and Mond [33] and Bector, Chandra and Husain [5] but this is, however, not pursued here.

The results of this paper also give duals to the (static) mathematical programming problems of Fletcher and Watson [18] (and some of its variants), which have not been reported in the literature explicitly. As a special case of this, we get the duality results of Mond [2], Mond and Schechter [23] and Watson [32].

Finally, a continuous fractional programming problem containing arbitrary norms is also considered and duality results are presented. These duality results generalize some results of Abrham and Buie [2] for the differentiable case and give a dynamic analogue of certain nondifferentiable fractional programming problems considered by Mond [20]. They also give duality results to the fractional analogues of problems considered by Watson [32], Fletcher and Watson [18], which have not been studied explicitly in the literature.

\section{Notations and statement of the problems}

Let $I=[a, b]$ be a real interval; let $f: I \times \mathbf{R}^{n} \times \mathbf{R}^{n} \rightarrow \mathbf{R}$ and $g: I \times \mathbf{R}^{n} \times \mathbf{R}^{n}$ $\rightarrow \mathbf{R}^{m}$ be continuously differentiable functions. In order to consider $f(t, x(t), \dot{x}(t))$, where $x: I \rightarrow \mathbf{R}^{n}$ is differentiable with derivative $\dot{x}$, denote the partial derivatives of $f$ by

$$
f_{t}, f_{1}=\left[\frac{\partial f}{\partial x_{1}}, \ldots, \frac{\partial f}{\partial x_{n}}\right], f_{2}=\left[\frac{\partial f}{\partial \dot{x}_{1}}, \ldots, \frac{\partial f}{\partial \dot{x}_{n}}\right] .
$$

The partial derivatives of $g$ are similarly written, using matrices with $m$ rows instead of one. Let $\mathbf{R}_{+}=[0, \infty)$; let $\mathbf{R}_{+}^{n}$ be the nonnegative orthant of $\mathbf{R}^{n}$. Let $C\left(I, \mathbf{R}^{m}\right)$ denote the space of continuous functions $\phi: I \rightarrow \mathbf{R}^{n}$, with the uniform norm; let $C_{+}\left(I, \mathbf{R}^{m}\right)$ denote the cone of non-negative functions in $C\left(I, \mathbf{R}^{m}\right)$. Let $X$ 
denote the space of piecewise smooth functions $x: I \rightarrow \mathbf{R}^{n}$, with the norm $\|x\|=\|x\|_{\infty}+\|D x\|_{\infty}$, where the differentiation operation $D$ is given by

$$
y=D x \Leftrightarrow x(t)=\alpha+\int_{a}^{t} y(s) d s,
$$

and $x(a)=\alpha, x(b)=\beta$ are given boundary values. (Thus $D=d / d t$ expept at discontinuities.) Denote by $V$ the space of piecewise smooth functions $\lambda: I \rightarrow \mathbf{R}^{m}$ (written as column vectors). Superscript $T$ denotes matrix transpose.

For each $t \in I$, let $P(t)$ and $Q_{j}(t)(j=1,2, \ldots, m)$ be $p \times m$ and $q \times n$ matrices respectively, with $P(\cdot)$ and $Q_{j}(\cdot)$ continuous on $I$. The norms $\|\cdot\|_{A}$ and $\|\cdot\|_{B(j)}$ are arbitrary norms in their appropriate spaces; $\|\cdot\|_{A}^{*}$ denotes the dual norm to $\|\cdot\|_{A}$. The generalized Cauchy inequality (Fletcher and Watson [18]) states that $x^{T} u \leqslant\|x\|\|u\|^{*}$. Following the notation in [14], $\|Q .(t) x(t)\|_{B .}$ shall denote the vector whose $j$ th component is $\left\|Q_{j}(t) x(t)\right\|_{B(j)}$. By convention, let $w_{(}(t)^{T} q .(t)$ denote the matrix whose $j$ th row is $w_{j}(t)^{T} Q_{j}(t)$, for $j=1,2, \ldots, m$.

Let $S \subset \mathbf{R}^{m}$ be a convex cone, with nonempty interior. For the definitions of $S$-convex functions and $S^{*}$-nearly convex functions, we shall refer to [13, 15]. If $K$ is a convex cone, its dual cone $K^{*}$ is the set of continuous linear functionals mapping $K$ into $\mathbf{R}_{+}$.

The primal and dual problems discussed are the following:

(P): Minimize $\Phi(x)=\int_{I}\left[f(t, x(t), \dot{x}(t))+\|P(t) x(t)\|_{A}\right] d t$

$$
\begin{gathered}
\text { subject to } x(a)=\alpha, \quad x(b)=\beta, \\
g(t, x(t), \dot{x}(t))+\|Q .(t) x(t)\|_{B} \in-S,
\end{gathered}
$$

(D): Maximize $\Psi(u, z, \lambda, w)$

$$
\begin{aligned}
=\int_{I} & {\left[f(t, u(t), \dot{u}(t))+u(t)^{T} P(t) z(t)\right.} \\
& \left.+\lambda(t)^{T}\left[g(t, u(t), \dot{u}(t))+w .(t)^{T} Q .(t) u(t)\right]\right] d t
\end{aligned}
$$

subject to $u(a)=0, u(b)=0$,

$$
f_{1}(t, u(t), \dot{u}(t))+z(t)^{T} P(t)+\lambda(t)^{T}\left[g_{1}(t, u(t), \dot{u}(t))+w .(t)^{T} Q .(t)\right]
$$

$$
-D\left[f_{2}(t, u(t), \dot{u}(t))+\lambda(t)^{T} g_{2}(t, u(t), \dot{u}(t))\right]=0,
$$

$$
\|z(t)\|_{A}^{*} \leqslant 1, \quad\left\|w_{j}(t)\right\|_{B(j)}^{*} \leqslant 1 \quad(j=1,2, \ldots, m), \lambda(t) \in S^{*}(t \in I) .
$$

In the following theorems, it will further be assumed that $G(x)(t):=$

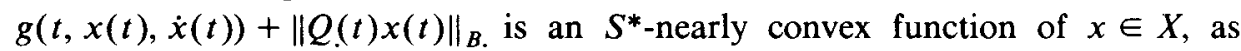
defined in [15]. If, in particular, $S=\mathbf{R}_{+}^{m}$, then $G$ is the sum of a (Fréchet) differentiable function of $x$ and an $S$-convex function of $x$, hence $G$ is $S^{*}$-nearly convex as required. 
If $P(\cdot)=0$ and each $Q_{j}(\cdot)=0$, then (P) and (D) reduce to the pair of variational problems considered by Mond and Hanson [22]. If $Q_{j}(\cdot)=0$ and $\|\cdot\|_{A}$ is the $L^{2}$-norm, the results of [6] are obtained with $B(t)=P(t)^{T} P(t)$. However, the present method of proving converse duality differs from that of [6]. The present method assumes a solvability hypothesis, rather than using the Fritz John conditions, and thus does not require second order derivatives of $f$ and $g$, as [6] required.

It is convenient, as in $[13,6]$, to shift the origin in $X$ to make the boundary conditions $x(a)=0=x(b)$; this is assumed in the proof; the original problem is recovered by a shift of origin.

\section{Conditions necessary or sufficient for optimality}

THEOREM 1 (F. John conditions). If $(\mathrm{P})$ attains a local minimum at $x=x_{0} \in X$, then there exist Lagrange multipliers $\tau \in \mathbf{R}_{+}$and piecewise smooth $\lambda: I \rightarrow S^{*}, z$ : $I \rightarrow \mathbf{R}^{p}, w_{j}: I \rightarrow \mathbf{R}^{q_{j}}(j=1,2, \ldots, m)$, with $\tau$ and $\lambda$ not both zero, such that

$$
\begin{gathered}
\tau\left[f_{1}\left(t, x_{0}(t), \dot{x}_{0}(t)\right)+z(t)^{T} P(t)\right] \\
+\lambda(t)^{T}\left[g_{1}\left(t, x_{0}(t), \dot{x}_{0}(t)\right)+w .(t)^{T} Q .(t)\right] ; \\
-D\left[\tau f_{2}\left(t, x_{0}(t), \dot{x}_{0}(t)\right)+\lambda(t)^{T} g_{2}\left(t, x_{0}(t), \dot{x}_{0}(t)\right)\right]=0 ; \\
\lambda(t)^{T}\left[g\left(t, x_{0}(t), \dot{x}_{0}(t)\right)+w .(t)^{T} Q .(t) x_{0}(t)\right]=0 ; \\
\|z(t)\|_{A}^{*} \leqslant 1 ; \quad\left\|w_{j}(t)\right\|_{B(j)}^{*} \leqslant 1 \quad(j=1,2, \ldots, m) ; \\
x_{0}(t)^{T} P(t) z(t)=\left\|P(t) x_{0}(t)\right\|_{A} ; \\
x_{0}(t)^{T} Q_{j}(t) w_{j}(t)=\left\|Q_{j}(t) x_{0}(t)\right\|_{B(j)} \quad(j=1,2, \ldots, m) ;
\end{gathered}
$$

holds for all $t \in I$, where $w(t) \equiv\left\{w_{j}(t): j=1,2, \ldots, m\right\}$.

Conversely, if (10) to (14) hold with $\tau=1$ and $x_{0}(\cdot)$ feasible for $(\mathrm{P})$, and if $f(t, \cdot, \cdot)$ is convex and $g(t, \cdot, \cdot)+\|Q(\cdot) x(\cdot)\|_{B \cdot}$ is $S$-convex for each $t \in I$, then $x_{0}$ minimizes $(\mathrm{P})$.

Proor. The problem (P) may be expressed as (EP):

$$
\text { Minimize } \Phi(x)=F(x)+J(x) \text { subject to } G(x) \in-K,
$$

in which

$$
F(x)=\int_{I} f(t, x(t), \dot{x}(t)) d t, \quad J(x)=\int_{I}\|P(t) x(t)\|_{A} d t,
$$




$$
\begin{aligned}
& G: X \rightarrow C\left(I, \mathbf{R}^{m}\right) \text { is given by }(\forall t \in I, x \in X) \\
& G(x)(t)=g(t, x(t), \dot{x}(t))+\|Q .(t) x(t)\|_{B .},
\end{aligned}
$$

and the convex cone $K=\left\{y \in C\left(I, \mathbf{R}^{m}\right):(\forall t \in I) y(t) \in S\right\}$. From [15, Theorem 3], the Fritz John necessary conditions for (EP) to attain a local minimum at $x=x_{0}$ are the existence of Lagrange multipliers $\tau \in \mathbf{R}_{+}$and $\rho \in K^{*}$, not both zero, satisfying

$$
0 \in \tau \partial \Phi\left(x_{0}\right)+\partial(\rho G)\left(x_{0}\right) ; \quad 0=\rho G\left(x_{0}\right) .
$$

The cited theorem requires certain convex sets to be weak * compact; this holds for $(\mathrm{P})$. Here $\partial(\rho G)\left(x_{0}\right)$ denotes a subgradient for nearly convex functions (see [15]).

Since $f(t, \cdot, \cdot)$ is continuously differentiable, $F^{\prime}\left(x_{0}\right)$ is given ([13], page 16) by (19)

$(\forall v \in X) F^{\prime}\left(x_{0}\right) v=\int_{I}\left[f_{1}\left(t, x_{0}(t), \dot{x}_{0}(t)\right) v(t)+f_{2}\left(t, x_{0}(t), \dot{x}_{0}(t)\right) \dot{v}(t)\right] d t$.

Assume now, subject to later validation, that $\rho \in K^{*}$ can be represented by a measurable function $\lambda: I \rightarrow S^{*}$ satisfying

$$
\left(\forall \zeta \in C\left(I, \mathbf{R}^{m}\right)\right) \quad \rho \zeta=\int_{I} \lambda(t)^{T} \zeta(t) d t .
$$

Let $\xi(t, x)=\|Q(t) x(t)\|_{B,}$, for $x \in X, t \in I$. Then

$$
\rho \xi(\cdot, x)=\int_{I} \lambda(t)^{T} \xi(t, x) d t .
$$

Denote by $\partial_{C}$ the Clarke generalized subgradient [7] with respect to $x$. Then

$$
\begin{aligned}
\partial_{C}\left(\lambda(t)^{T} \xi(t, x)\right) & \subset \sum_{j=1}^{m} \partial_{C}\left(\lambda_{j}(t) \xi_{j}(t, x)\right) \quad \text { by [7, Proposition 8] } \\
& =\sum_{j=1}^{m}\left|\lambda_{j}(t)\right| \partial_{C}\left(\operatorname{sgn}\left(\lambda_{j}(t)\right) \xi_{j}(t, x)\right) \\
& =\sum_{j=1}^{m}\left|\lambda_{j}(t)\right| \operatorname{sgn}\left(\lambda_{j}(t)\right) \partial_{C}\left(\xi_{j}(t, x)\right)
\end{aligned}
$$

using the representation [7] of $\partial_{C}(\cdot)$ as the convex hull of limit points of gradients at smooth points near $x ; \Sigma$ denotes here algebraic sum of sets. From Watson [32], since $\xi_{j}(t, \cdot) \cdot$ is convex,

$$
\begin{aligned}
\partial_{C} \xi_{j}(t, x)=\partial \xi_{j}(t, x)=\left\{w_{j}(t)^{T} Q_{j}(t): w_{j}(t)\right. & \in \mathbf{R},\left\|w_{j}(t)\right\|_{B(j)}^{*} \leqslant 1, \\
& \left.\xi_{j}(t, x)=w_{j}(t)^{T} Q_{j}(t) x\right\} .
\end{aligned}
$$


From [10], it follows that $q \in \partial(\rho \xi)(\cdot, x)$ if and only if there exists a measurable function $\eta: I \rightarrow \mathbf{R}^{m \times n}$ such that

$$
(\forall t \in I) \eta(t) \in \partial_{C} \xi(t, x(t)) ; \quad(\forall v \in X) q v=\int_{I} \lambda(t)^{T} \eta(t) v(t) d t .
$$

Here, from (22) and (23), $\eta(t)=w(t)^{T} Q(t)$. Therefore

$$
\begin{aligned}
\partial(\rho G)\left(x_{0}\right) v & :=\left\{\theta v: \theta \in \partial(\rho G)\left(x_{0}\right)\right\} \\
\subset \int_{I} \lambda(t)^{T}\left[g_{1}\left(t, x_{0}(t), \dot{x}_{0}(t)\right) v(t)+g_{2}\left(t, x_{0}(t), \dot{x}_{0}(t)\right) \dot{v}(t)\right. & \left.+\left(w \cdot(t)^{T} Q .(t)\right) v(t)\right] d t .
\end{aligned}
$$

Using (18), (19) and (24), necessary conditions for a minimum of (P) at $x_{0}$ are that $\tau \geqslant 0$ and $\lambda(\cdot)$ and $w(\cdot)$ exist, satisfying conditions of (23), and also $z$ : $I \rightarrow \mathbf{R}^{n}$ satisfying the similar conditions $\|z(t)\|_{A}^{*} \leqslant 1, \quad z(t)^{T} P(t) x_{0}(t)=$ $\left\|P(t) x_{0}(t)\right\|_{A}$ such that, for each $v \in X$,

$$
\begin{gathered}
\int_{I}[M(t) v(t)+N(t) \dot{v}(t)] d t=0 ; \\
\int_{I} \lambda(t)^{T}\left[g\left(t, x_{0}(t), \dot{x}_{0}(t)\right)+\left\|Q .(t) x_{0}(t)\right\|_{B}\right] d t=0,
\end{gathered}
$$

where

$$
\begin{aligned}
M(t)= & \tau f_{1}\left(t, x_{0}(t), \dot{x}_{0}(t)\right)+z(t)^{T} P(t) \\
& +\lambda(t)^{T}\left[g_{1}\left(t, x_{0}(t), \dot{x}_{0}(t)\right)+w .(t)^{T} Q .(t)\right] ; \\
N(t)= & \tau f_{2}\left(t, x_{0}(t), \dot{x}_{0}(t)\right)+\lambda(t)^{T} g_{2}\left(t, x_{0}(t), \dot{x}_{0}(t)\right) .
\end{aligned}
$$

Integrating the first part of (25) by parts, and using the boundary condition $v(a)=v(b)=0$, leads to $\int_{I}[N(t)-H(t)] \dot{v}(t) d t=0$, where $H$ is an indefinite integral of $M$. This holds whenever $\dot{v}$ is replaced by a piecewise continuous function $\sigma$ for which $\int_{I} \sigma(t) d t=0$. Using [11, page 500, Lemma 2] it follows that $N(\cdot)-H(\cdot)$ is constant almost everywhere. (The cited lemma assumes $N-H$ piecewise continuous, but extends readily to measurable.) Hence, for almost all $t$, $N(t)$ is differentiable, and $N=M$. This proves (10) for almost all $t$. Also (11) follows from the second part of (18), with (14).

In order to validate the representation of $\rho$ by a function $\lambda(\cdot)$, note that the proof leading to (10) and (11) remains valid, without this assumption, if $\lambda(\cdot)$ is considered as a Schwartz distribution. However, (10) and (11) is a first order linear ordinary differential equation system for $\lambda(\cdot)$, given $x_{0}, z$ and $w$, and so possesses a piecewise smooth solution $\lambda(\cdot)$. Then $z$ and $w$ are also piecewise smooth, from (10) and (11). Hence $N-H$ is constant for all $t$. 
The sufficient conditions for a minimum follow immediately [13, page 64], since $(\mathrm{P})$ is then a convex problem.

The local minimum $x_{0}$ of (P) may be called normal if $\tau=1$, so that the Fritz John conditions (10) to (14) reduce to Kuhn-Tucker conditions. Sufficient conditions for $x_{0}$ to be normal are the Slater conditions, either (i) that $(\mathrm{P})$ is a convex problem and, for some $\xi \in X, G(\xi) \in-$ int $S$, or (ii) that, for some $q \in X$, the directional derivative of $G$ at $x_{0}$ in direction $q$ satisfies $G^{\prime}\left(x_{0} ; q\right) \in-$ int $S$. The proof of (i) is standard; for (ii), if $\tau=0$ then $0 \neq \rho \in S^{*}$, hence $\rho G^{\prime}\left(x_{0} ; q\right)<0$, contradicting $\rho G^{\prime}\left(x_{0} ; q\right)=0$ from (18).

\section{Duality and converse duality}

Using (15), problem (P) is equivalently written as the problem (EP):

Minimize $\Phi(x)$ subject to $G(x) \in-K$

Since $g(t, x(t))+\|Q(t) x(t)\|_{B}$, has been assumed to be an $S^{*}$-nearly convex function of $x \in X, G$ is $K^{*}$-nearly convex. Assume now that $G$ is $K$-convex; this follows if $S=\mathbf{R}_{+}^{m}$ and each component $g_{i}(t, x(t))$ is a convex function of $x$. Then, using (18), the problem (D) is equivalently written as the problem (ED):

(27) Maximize $\Phi(u)+\rho G(u)$ subject to $\rho \in K^{*}, 0 \in \partial(\Phi+\rho G)(u)$.

Here $\Psi(u, z, \lambda, w)=\Phi(u)+\rho G(u) \equiv L(u, \rho)$, and $\partial L(u, \rho)$ means $\partial L(\cdot, \rho)$ at $u$.

THEOREM 2 (Duality). Let $f$ be convex, and let $G$ be $K$-convex. If $x$ is feasible for (P) and $(u, z, \lambda, w)$ is feasible for (D), then

$$
\Phi(x) \geqslant \Psi(u, z, \lambda, w) .
$$

If equality holds in (28), then $x$ optimizes (P) and ( $u, z, \lambda, w)$ optimizes (D). If $\bar{x}$ minimizes $(\mathrm{P})$ and $\tau=1$, then there exist $(\bar{z}, \bar{\lambda}, \bar{w})$ such that $(\bar{x}, \bar{z}, \bar{\lambda}, \bar{w})$ maximizes (D), and $\boldsymbol{\Phi}(\bar{x})=\Psi(\bar{x}, \bar{z}, \bar{\lambda}, \bar{w})$.

Proof. The proof of $(28)$ is immediate, since $(\mathrm{P})$ is a convex problem. Theorem 1 gives $(\bar{z}, \bar{\lambda}, \bar{w})$ with the stated properties.

Here, as with a differentiable problem, $\tau=1$ holds under a suitable constraint qualification, such as Slater's.

For proving converse duality, the following solvability hypothesis will be assumed (with $(\bar{u}, \bar{\rho})$ denoting the optimum for (ED)).

(H): Whenever $\bar{\rho}+d \in K^{*}$ with $\|d\|$ sufficiently small, there exists a solution $u=\bar{u}+\theta(d)$ to $0 \in \partial(\Phi+(\bar{\rho}+d) G)(u)$ for which $\theta(0)=0$ and $\theta(\cdot)$ is continuous. 
THEOREM 3 (Converse Duality). Let $f$ be convex, and let $G$ be $K$-convex. If $(\bar{u}, \bar{\rho})$ maximizes (ED) and $(\mathrm{H})$ holds then $\bar{u}$ minimizes $(\mathrm{EP})$, and $\Phi(u)=L(\bar{u}, \bar{\rho})$.

Proof. Since $(\bar{u}, \bar{\rho})$ maximizes (ED), we have

$$
[\Phi(\bar{u}+\theta(d))-(\bar{\rho}+d) G(\bar{u}+\theta(d))]-[\Phi(\bar{u})+\bar{\rho} G(\bar{u})] \leqslant 0,
$$
i.e.,

$$
[L(\bar{u}+\theta(d), \bar{\rho})-L(\bar{u}, \bar{\rho})+d G(\bar{u}+\theta(d))] \leqslant 0 .
$$

But $0 \in \partial L(\bar{u}, \bar{\rho})$ is a constraint in (ED) and hence, by definition of subgradient, $L(\bar{u}+\theta(d), \bar{\rho})-L(\bar{u}, \bar{\rho}) \geqslant 0$. Therefore by $(29), d G(\bar{u}+\theta(d)) \leqslant 0$. This inequality also holds with $d$ replaced by $\alpha d$ for $0<\alpha \leqslant 1$. Now letting $\alpha \downarrow 0$, we get $\theta(\alpha d) \rightarrow 0$ and then using the fact that $0<\alpha \leqslant 1$, we obtain $d G(\bar{u}) \leqslant 0$. Thus $d \in K^{*}$ and $G(\bar{u}) \in-K$.

Now setting $d=-\frac{1}{2} \bar{\rho}$, we observe that $\bar{\rho}+\alpha d \in K^{*}$ for $0<\alpha \leqslant 1$. Hence $-\frac{1}{2} \bar{\rho} G(\bar{u}) \leqslant 0$, i.e., $\bar{\rho} G(\bar{u}) \geqslant 0$. But $\bar{\rho} G(\bar{u}) \leqslant 0$ as $G(\bar{u}) \in-K$ and $\rho \in K^{*}$. Therefore $\bar{\rho} G(\bar{u})=0$ and consequently $\Phi(\bar{u})=L(\bar{u}, \bar{\rho})$. The result then follows by Theorem 2.

\section{Related problems}

As in [22] and [6], these duality results can be extended to the corresponding problem (P1), omitting the boundary conditions $x(a)+\alpha, x(b)=\beta$, and (D1) with "natural boundary values". Thus (D1) is the problem (D) together with the additional boundary conditions

$$
f_{2}(t, x(t), \dot{x}(t))-\lambda(t)^{T} g_{2}(t, x(t), \dot{x}(t))=0,
$$

whenever $t=a$ and $t=b$. The boundary conditions (30) are similar to "natural boundary conditions" in the calculus of variations [12].

\section{Certain static cases}

If (P1) and (D1) are independent of $t$, then they reduce to the following nondifferentiable mathematical programming problems:

Primal (P2): Minimize $f(x)+\|P x\|_{A}$ subject to $g(x)+\|Q . x\|_{B .} \in-S$.

$$
\text { Maximize } f(x)+x^{T} P z+\lambda^{T}\left(g(x)+w^{T} Q x\right) \text { subject to }
$$

Dual (D2): $\quad \nabla f(x)+z^{T} P+\lambda^{T}\left[\nabla g(x)+w_{l}^{T} Q.\right]=0, \quad\|z\|_{A}^{*} \leqslant 1, \lambda \in S^{*}$,

$$
\left\|w_{j}\right\|_{B(j)}^{*} \leqslant 1 \quad(j=1,2, \ldots, m) .
$$


Here, it is noted that the primal-dual pair (P2)-(D2) has not been explicitly reported in the literature. In case $Q_{j}=0$ for all $j$ and $S=\mathbf{R}_{+}^{m}$, (P2) and (D2) reduce to the primal-dual pair studied by Watson [32] which, in turn, includes the problems of Mond [21] and Mond and Schechter [23] as special cases. The pair (P2)-(D2) could also be considered as an extension of Schechter [31] with regard to the converse duality, because in [31], it is given for differentiable constraints only. Also by taking $m=1, S=\mathbf{R}_{+}, g(x)=-\delta,(\delta>0)$ (P2) reduces to the problem of Fletcher and Watson [18], who give optimality conditions only and do not discuss duality.

\section{A fractional analogue}

Taking $h: I \times \mathbf{R}^{n} \times \mathbf{R}^{n} \rightarrow \mathbf{R}$, similar to $f$, and $E(t)$ an $(n \times l)$ matrix with $E(\cdot)$ continuous for each $t \in I$, the following fractional analogue of problem (P) is considered.

Primal (FP):

$$
\text { Minimize } \frac{\int_{b}^{a}\left[f(t, x(t), \dot{x}(t))+\|P(t) x(t)\| A_{1}\right] d t}{\int_{b}^{a}\left[h(t, x(t), \dot{x}(t))-\|E(t) x(t)\|_{A_{2}}\right] d t}
$$

$$
\begin{gathered}
\text { subject to } \quad x(a)=\alpha, \quad x(b)=\beta \\
g(t, x(t), \dot{x}(t))+\|Q .(t) x(t)\|_{B .} \in-S,
\end{gathered}
$$

where over the feasible region of (FP), the denominator of the objective function is strictly positive and the numerator is non-negative. Also $f(t, \cdot, \cdot)$ and $-h(t, \cdot, \cdot)$ are convex functions for each $t \in I$ and $g(t, \cdot, \cdot)+\|Q .(t)(\cdot)\|_{B .}$ is $S$-convex. Other symbols have the same meanings as in Section 2.

Using an abstract version of Dinkelbach's [17] result, given by Craven and Mond [16], and following techniques similar to Bector, Chandra and Gulati [4] and Jagannathan [19], the following dual problem (FD) is constructed:

Dual (FD):

\section{Maximize $\mu$}

$$
\text { subject to } u(a)=\alpha, \quad u(b)=\beta,
$$

$$
\begin{aligned}
& {\left[f_{1}(t, u(t), \dot{u}(t))+z(t)^{T} P(t)-\mu h_{1}(t, u(t), \dot{u}(t))+\mu \sigma(t)^{T} E(t)\right]} \\
& +\lambda(t)^{T}\left[g_{1}(t, u(t), \dot{u}(t))+w \cdot(t)^{T} Q .(t)\right] \\
& -D\left[f_{2}(t, u(t), \dot{u}(t))-\mu h_{2}(t, u(t), \dot{u}(t))+\lambda(t)^{T} g_{2}(t, u(t), \dot{u}(t))\right]=0, \\
& \int_{a}^{b}\left[f(t, u(t), \dot{u}(t))-\mu h(t, u(t), \dot{u}(t))+u(t)^{T} P(t) z(t)\right. \\
& \left.-\mu u(t)^{T} E(t) \sigma(t)+\lambda(t)^{T}\left(g(t, u(t), \dot{u}(t))+w .(t)^{T} Q .(t) u(t)\right)\right] d t=0,
\end{aligned}
$$




$$
\begin{gathered}
\|z(t)\|_{A_{1}}^{*} \leqslant 1, \quad\|\sigma(t)\|_{A_{2}}^{*} \leqslant 1, \\
\left\|w_{j}(t)\right\|_{B(j)}^{*} \leqslant 1 \quad(j=1,2, \ldots, m), \\
\lambda(t) \in S^{*}(t \in I), \quad \mu \geqslant 0 .
\end{gathered}
$$

The duality results between (FP) and (FD) follow essentially on the lines of Theorems 2 and 3 and certain obvious modifications, similar to [4] and [19]. Also for $P(t)=0, Q_{j}(t)=0, E(t)=0$ for all $t \in I,(\mathrm{FP})$ and (FD) reduce to certain differentiable fractional continuous programs of [2].

If (FP) and (FD) are independent of $t$, a fractional analogue of (P2) together with its dual will be obtained. Such fractional problems have not been explicitly studied in the fractional programming literature. As a very special case, if $Q_{j}=0$ for all $j,\|\cdot\|_{A_{1}}$ and $\|\cdot\|_{A_{2}}$ are $L^{2}$-norms and $S=\mathbf{R}_{+}^{m}$, the fractional problem studied by Mond [20] is derived.

\section{Acknowledgment}

One of the authors (S. Chandra) acknowledges the financial support and excellent research facilities provided by the authorities of the University of Melbourne during his term of a visiting research fellowship.

\section{References}

[1] J. Abrham and R. N. Buie, 'Kuhn-Tucker conditions and duality in continuous programming', Utilitas Math. 16 (1979), 15-37.

[2] J. Abrham and R. N. Buie, 'Duality in continuous fractional programming', Utilitas Math. 17 (1980), 35-44.

[3] J. Abrham and R. N. Buie, 'Duality in continuous programming and optimal control', Utilitas Math. 17 (1980), 103-109.

[4] C. R. Bector, S. Chandra and T. R. Gulati, 'Duality for fractional control problems', Proc. Fifth Manitoba Conference on Numerical Math., (1975), 231-241.

[5] C. R. Bector, S. Chandra and I. Husian, 'Generalized concavity and duality in continuous programming', communicated to Utilitas Math.

[6] S. Chandra, B. D. Craven and I. Husian, 'A class of nondifferentiable continuous programming problems', J. Math. Anal. Appl, to appear

[7] F. H. Clarke, 'A new approach to Lagrange multipliers', Math. Oper. Res. 1 (1976), 165-174.

[8] F. H. Clarke, 'The maximum principle under minimal hypothesis', SIAM. J. Control Optim. 14 (1976), 1078-1091.

[9] F. H. Clarke, 'Inequality constraints in the calculus of variations', Canadian J. Math. 29 (1977), 528-540.

[10] F. H. Clarke, 'Generalized gradients of Lipschitz functionals', Advances in Math. 40 (1981), 52-67.

[11] R. Courant, Differential and integral calculus, vol. 2, Blackie, London and Edinburgh, 1936/1948.

[12] R. Courant and D. Hilbert, Methods of mathematical physics, vol. 1, Wiley Interscience, New York, 1948. 
[13] B. D. Craven, Mathematical programming and control theory, Chapman \& Hall, London, 1978.

[14] B. D. Craven and B. Mond, 'Sufficient Fritz John conditions for nondifferentiable convex programming', J. Austral. Math. Soc., Ser. B, 19 (1976), 462-468.

[15] B. D. Craven and B. Mond, 'Lagrangian conditions for quasi-differentiable optimization', Proc. of 9th Int. Conf. on Mathematical Programming, Budapest 1976, A. Prékopa (ed.), vol. 1, 177-192, in Survey of mathematical programming, Akadémiai Kiado, Budapest and North-Holland, Amsterdam, 1979.

[16] B. D. Craven and B. Mond, 'Continuous nonlinear fractional programming', unpublished.

[17] W. Dinkelbach, 'On nonlinear fractional programming', Management Sci. 13 (1967), 492-498.

[18] R. Fletcher and G. A. Watson, 'First and second order conditions for a class of nondifferentiable optimization problems', Math. Programming 18 (1980), 291-307.

[19] R. Jagannathan, 'Duality for nonlinear fractional programs', Z. Oper. Res. 17 (1973), 1-3.

[20] B. Mond, 'A class of nondifferentiable fractional programming problems', ZAMM 58 (1978), 337-341.

[21] B. Mond, 'A class of nondifferentiable mathematical programming problems', J. Math. Anal. Appl. 46 (1974), 169-174.

[22] B. Mond and M. A. Hanson, 'Duality for variational problems', J. Math. Anal. Appl. 18 (1967), $355-364$.

[23] B. Mond and $M$. Schechter, 'A programming problem with an $L_{p}$ norm in the objective function', J. Austral. Math. Soc. (Ser. B) 19 (1976), 333-342.

[24] B. Mond and T. Weir, 'Generalized concavity and duality', Generalized concavity in optimization and economics, S. Schaible and W. T. Ziemba (ed.), Academic Press, New York, 1981.

[25] T. W. Reiland, 'Optimality conditions and duality in continuous programs', J. Math. Anal. Appl. 77 (1980), 329-343.

[26] T. W. Reiland and M. A. Hanson, 'Generalized Kuhn-Tucker conditions and duality for continuous nonlinear programming problems', J. Math. Anal. Appl. 74 (1980), 578-598.

[27] S. M. Robinson, 'Stability theory for systems of inequalities, part II: Differentiable nonlinear systems', SIAM J. Numer. Anal. 13 (1976), 476-513.

[28] R. T. Rockafellar, 'Conjugate convex functions in optimal control and the calculus of variations', J. Math. Anal. Appl. 32 (1970), 174-222.

[29] R. T. Rockafellar, 'Existence and duality theorems for convex problems of Bolza', Trans. Amer. Math. Soc. 159 (1971), 1-40.

[30] R. T. Rockafellar, 'Dual problems of optimal control', in Techniques of optimization, A. V. Balakrishnan (ed.), Academic Press, New York, 1972.

[31] M. Schechter, 'More on subgradient duality', J. Math. Anal. Appl. 71 (1979), 251-262.

[32] G. A. Watson, 'A class of programming problems whose objective function contains a norm', $J$. Math. Anal. Appl. 23 (1978), 401-411.

[33] T. Weir, M. A. Hanson and B. Mond, 'Generalized concavity and duality in continuous programming', Technical Report M649, Florida State University, Talahassee, Florida, Nov. 1982.

Mathematics Department

Indian Institute of Technology

Hauz Khas, New Delhi 110016

India

Mathematics Department

Regional Engineering College

Srinagar, Kashmir

India
Mathematics Department

University of Melbourne

Parkville, Victoria 3052

Australia 